\title{
A Hybrid Distance Map Based and Morphologic Thinning Algorithm
}

\author{
Klaus Donath, Matthias Wolf*, Radim Chrástek, and Heinrich Niemann \\ FORWISS, Knowledge Processing Research Group, Haberstr. 2, 91058 Erlangen, \\ donath@forwiss.de, \\ http://www-wv. informatik. uni-erlangen.de/ ${ }^{\sim}$ donath
}

\begin{abstract}
A lot of applications need that an object is transformed into its medial axis while preserving its topology. In this paper we present a thinning algorithm based on special masks preserving connectivity. The thinning process is controled by a distance map to overcome problems of a former approach. As a result we obtain a skeleton that has minor artefacts only and which is suitable in the field of blood vessel analysis. We are providing results on synthetic and real images that are compared with another approach.
\end{abstract}

\section{Introduction}

Many computer vision applications need characteristics of an object. Mainly elongated 2D objects are usually transformed into their $1 \mathrm{D}$ medial axis while preserving the topology. A point in a binary image whose deletion does not destroy the topology is called a simple point. Thus, a process deleting simple points is called a thinning algorithm. Typical fields of application are found in optical character recognition or medical image analysis. I.e. in blood vessel analysis the skeleton of the vessel system can be used for computing the length of all vessels.

A thinning algorithm is a method that transforms an object into a 1 pixel thin line in the middle of the object while preserving its topology. Such a method should meet the following requirements:

- The skeleton must preserve the topology of the original object,

- The end points of the object should be part of its skeleton,

- The skeleton should be situated in the middle of the object that means every skeleton point has the same distance from at least 2 object borders,

- Rotation invariance: Skeletonisation and object rotation should correspond. Due to spatial discretisation this should be approximately satiesfied for arbitrary rotations.

- Noise invariance: The skeleton should be insensitive to noise.

However, it is known that these requirements cannot be fulfilled simultaneously. In particular, it is difficult to distinguish a large artefact from a little feature.

\footnotetext{
* Now with: Siemens Medical Solutions USA, Inc., 51 Valley Stream Parkway, Malvern,
} PA 19355, USA 


\subsection{Thinning Algorithms}

In literature a large number of thinning algorithms can be found that meet these requirements more or less. Regarding their basic ideas they can be roughly divided into the following groups:

- Morphological methods: Those are local processes based on several masks typically of size $3 \times 3$ or $5 \times 5$. For that matter they are rather fast but due to the locality of small masks there are often artefacts remaining.

- Border tracking algorithms: For each border point a corresponding point at the opposite border is searched. The skeleton is found in the middle of the line connecting both points. Beginning with two corresponding border points the object borders are then tracked by using additional information, e.g. the direction. At last the skeleton points are connected. The resulting curve is rather smooth but concave objects or bifurcations can cause problems of finding corresponding pixels.

- Extractions from distance maps: The approach starts out from computing a distance map (DM) containing distances of object points to their closest object border according to a given metric. Then the skeleton is extracted from the DM by searching local maximums and connecting them to a closed curve.

There is no universal algorithm combining all advantages and meeting all requirements but for most applications a suitable skeleton algorithm can be found. In case of blood vessel analysis the number of skeleton points are assumed to correspond to the vessel length whereas the positions of skeleton points in the DM correspond to the radii of blood vessels. Therefore skeleton points should be identical with maximums of the DM.

In the following we will focus on morphological approaches. Usually, a morphological thinning algorithm is an iterative process. In a sequential approach a pixel is deleted in each iteration. Due to the locality of the data, only the values of some neighbours are necessary. Thus, an algorithm can be implemented as a parallel process which deletes a number of pixels in each iteration. The number of iterations itself depends on the maximum thickness of an object.

While earlier approaches mainly focused on the quality of the algorithm, nowadays there seems to be a focus on speed and complexity as well. Such an algorithm can be found in [3] published recently. This approach does not only give good results but it is also very fast and well founded. But unfortunately the requirement for 1 pixel thin midlines is not satisfied.

\section{Algorithm}

\subsection{Former Algorithm}

In [2] a morphological thinning algorithm is proposed for analyzing blood vessel images of the chicken yolk sack membrane. The method is an iterative conditional erosion based on 8 masks of size $3 \times 3$ (Fig. 1) adapted to thin elongated objects 

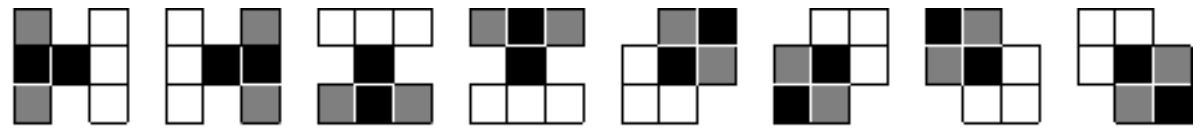

Fig. 1. Morphological thinning algorithm: $3 \times 3$ masks for conditional erosion.

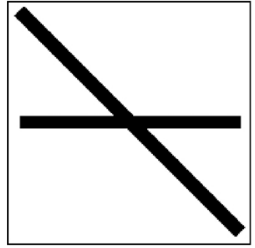

(a)

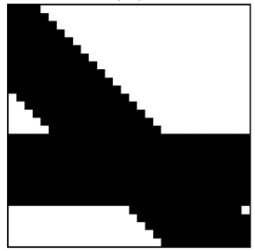

(e)

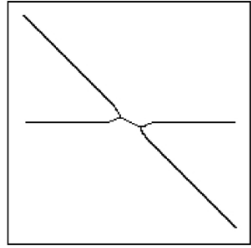

(b)

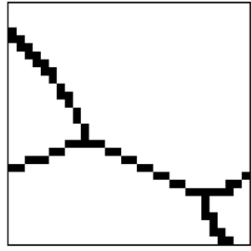

(f)

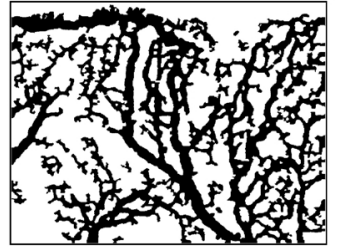

(c)

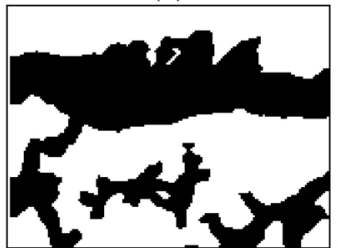

(g)

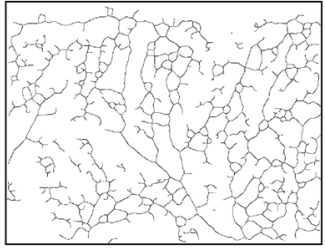

(d)

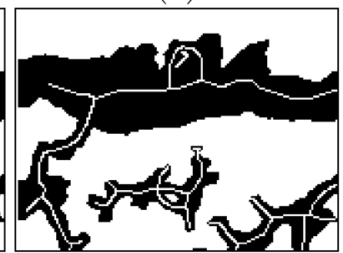

(h)

Fig. 2. Results of thinning method: (a) synthetic image and skeleton (b), (c) real image and skeleton $(\mathrm{d})$, and subimages (e, $\mathrm{f}, \mathrm{g}, \mathrm{h}),(\mathrm{h})$ skeleton is superimposed onto image.

preserving connectivity and with nearly no remaining artefacts. The approach uses an 8-topology for the object and a 4-topology for the background. Every iteration consists of 2 steps. At first, all object points which meet the condition of a mask are marked and in the second step all marked pixels are erased. These iterations are repeated until there are no more pixels to be erased.

An object point can be marked if at least one of its neighbours is an unmarked object point and the two neighbours on the left and on the right side are marked or unmarked object points and the 3 opposite points belong to the background (see Fig. 1).

In this way an object will be thinned to a 1 pixel thin line that might contain only little artefacts. The results are shown in Fig. 2.

\subsection{Problems}

In spite of the appearently good results the algorithm causes some problems. Because of the masks in Fig. 1 a single background pixel inside an object cannot be eroded thus yielding a skeleton which is not in the middle of the object. Furthermore the skeleton seems to be incorrect on image borders. Normally skeleton lines do not end on object borders but in a distance of a about vessel radius within the object. This is tolerable for vessels ending within an image but not for vessels on image borders (Fig. $2(\mathrm{~h})$ ). 

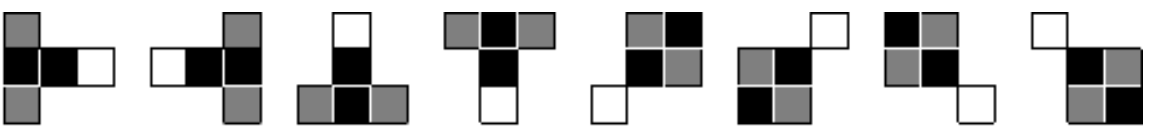

Fig. 3. Additional $3 \times 3$ masks that are used in the first iteration

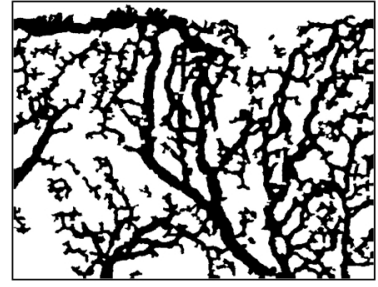

(a)

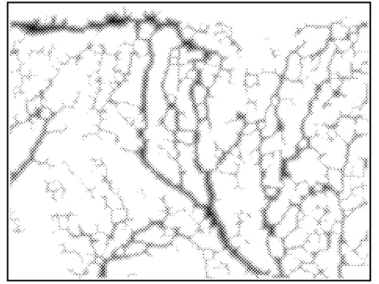

(b)

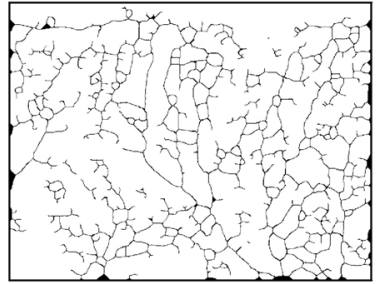

(c)

Fig. 4. Results of thinning method in combination with distance map: (a) binary image, (b) distance map and (c) interim skeleton image.

Sometimes the skeleton is not in the middle of the object. Caused by the used 8-topology the outer side of a curved object is much more eroded than the inner side. At last the produced skeleton has sometimes a 1 pixel thickness in terms of the 4-topology but tracking the skeleton is not always unambiguous in the 8-topology (Fig. 2 (f)). Due to this reason further thinning steps are necessary.

\subsection{Extensions of the New Algorithm}

To overcome the problems described above the algorithm needs to be enhanced. The problems at image borders are mainly caused by a 1 pixel frame of background points. Because it is a special effect of the segmentation process the frame is converted to object pixels and reconverted at the end of the thinning process.

To treat single background points inside an object in the first iteration special masks, shown in Fig. 3 are used. To reduce the object up to its midline the thinning method is combined with a distance map. A distance map can be approximated by an iterative erosion containing the distances of object pixels to their nearest borders. Using the 8-topology when eroding a binary image yields a maximum norm. An erosion based on the 4-topology gives distance values corresponding to the sum of the components of the distance vector. An Euclidian norm can be approximated by an erosion based on the 4-topology and the 8-topology alternately. The first step of the algorithm remains unchanged but at the second step only the marked points are erased that are not maximal in the 8-topology. In this way objects can be widely reduced to its midline. But plateaus in the distance map (that are often close to bifurcations) cause that small areas greater than 1 pixel remain. An example can be seen in Fig. 4 (c). Now the object has to be eroded with the known masks given in Fig. 1 ignoring the distance map until no more points can be deleted. The result of the method 


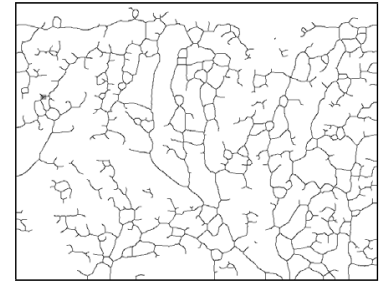

(a)

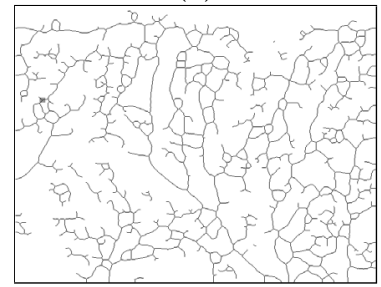

(c)

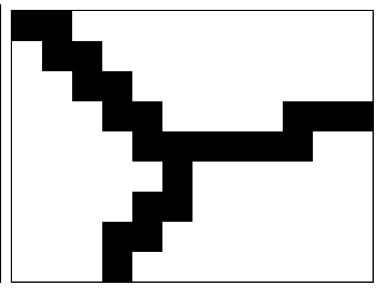

(b)

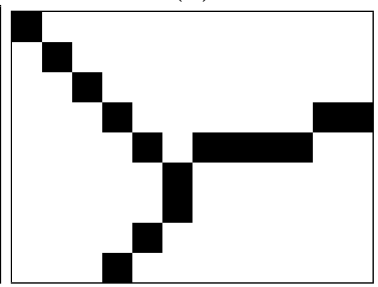

(d)

Fig. 5. Results of thinning method after erosion conditioned by distance map and after erosion without respecting distance map (a), subimage (b), (c) result after erasion of all simple points, (d) subimage of (c).
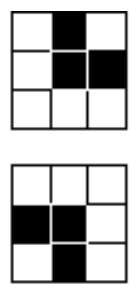

(a)
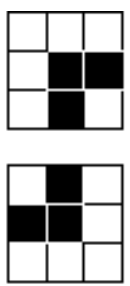
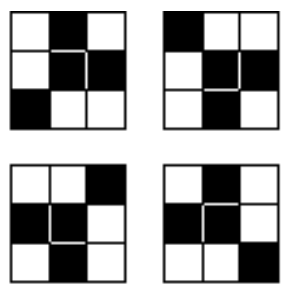

(b)

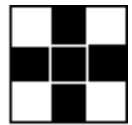

(c)

Fig. 6. The $3 \times 3$ masks that are used to find redundant points: (a) The points in the middle are redundant, (b) and (c) the points in the middle belong to a bifurcation and are not redundant.

up to this point is a skeleton of the object similar to that of the former method, the difference though is that the skeleton is positioned in the middle of the object. In Fig. 5 (a) the result is presented. In detail one can see that sometimes the skeleton is 1 pixel thin in the 4-topology but more than 1 pixel thin in the 8-topology (Fig. $5(\mathrm{~b})$ ). That means that much more simple points exist that have to be deleted.

For finding and deleting these simple points another set of masks is used. All points that match the condition of the masks in Fig. 6 (a) are simple points and can be erased while points that match a mask of Fig. 6 (b) or Fig. 6 (c) belong to a bifurcation of the skeleton and are not simple points. These points are not to be deleted. After erasion of all simple points there is a nearly perfect skeleton of an object whose lines are well positioned in the middle of the object, as well 


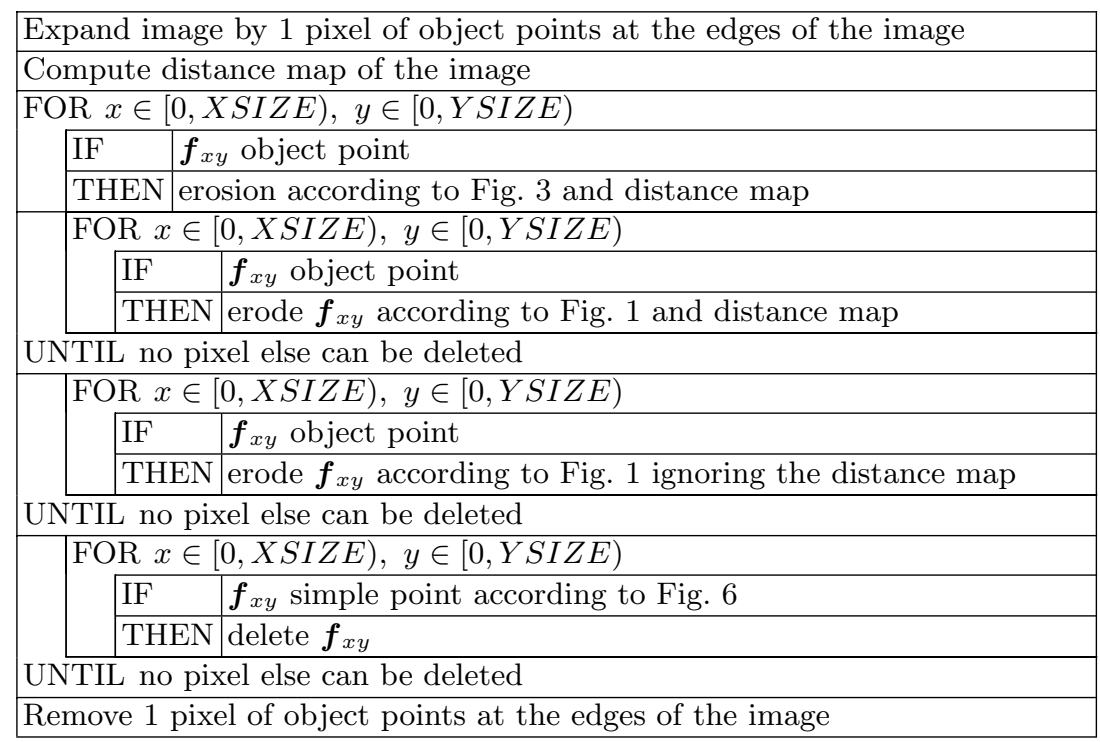

Fig. 7. Structure of the new thinning algorithm

as the objects endings are preserved in the skeleton and the skeleton is poor in artefacts. The final result is shown in Fig. 5 (c) as a whole and in Fig. 5 (d) in detail. Fig. 7 gives a structural overview of the new thinning algorithm.

\section{Results}

The results in Fig. 5 demonstrate that the new method yields a skeleton that preserves the topology of the object. In addition it is rather smooth, poor in artefacts and it is 1 pixel thin in terms of the 8-topology. The requirement on thinning methods to preserve end points of an object should form Y-like skeleton endings. Instead of this the skeleton ends in a single line at a distance of about a vessel radius from the vessel border. This is an advantage in analysing objects like blood vessels.

To measure the robustness of the method to rotation a synthetic image has been rotated by 45 and 90 degrees and the resulting images have been thinned too. As one can see in Fig. 8 our thinning method is invariant to rotation by 45 degrees. The different course of the skeleton at the lower end of skeleton in Fig. 8 (e) is caused by border noise originated in the rotation process. It also demonstrates that border noise can locally modify the course of the skeleton.

In the next step the results of our approach are compared to a method described in [5]. Fig. 9 shows the different results of the two methods. In most cases the position of the skeleton points differ about 1 or 2 pixels. Only skeleton points close to bifurcations can differ more caused by plateaus in the distance map. In case of blood vessel images the differences in skeletons are as low as in 


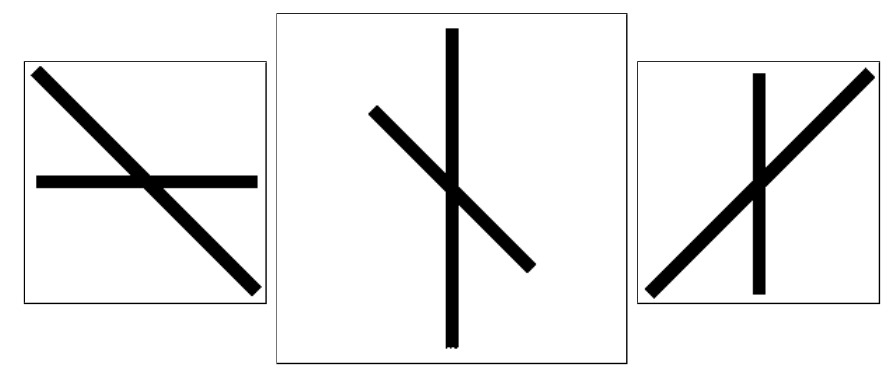

(a)

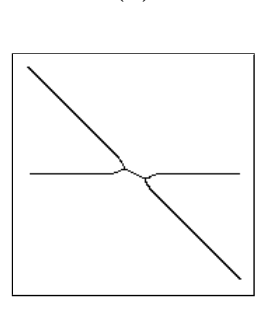

(d) (b)

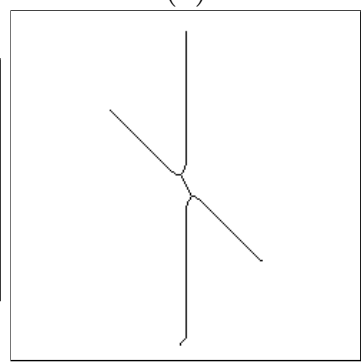

(e) (c)

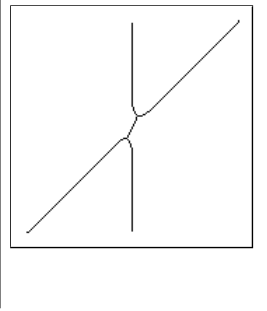

(f)

Fig. 8. Influence of rotation to the thinning method: (a) synthetic image, (b) image after beeing rotated by 45 degrees, (c) image after rotation by 90 degrees, (d), (e), (f) thinning results of (a), (b), (c).

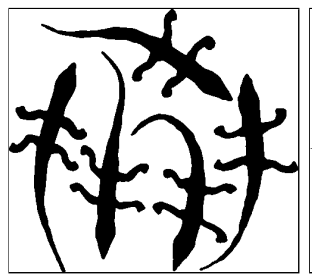

(a)

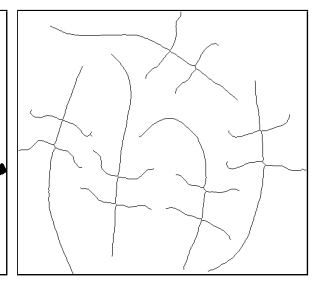

(b)

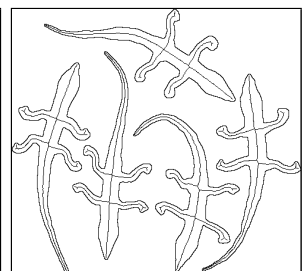

(c)

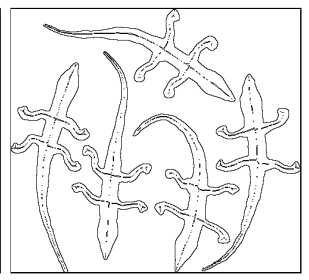

(d)

Fig. 9. Comparison of thinning methods: (a) image (from [5]), (b) result of mask based method, (c) result of [5], (d) comparison of (b) and (c) (identical points invisible)

the example above (Fig. 10). In addition the result of our proposed approach contains less artefacts than the skeleton of [5].

The processing time depends quadratically on the image size and the number of object points. At every iteration it is first tested whether a point is an object point or not. Then, the mask operation is processed only on object points. Because of local processing the method is rather fast. A typical blood vessel image of size $752 \times 576$ and nearly 40 percent object pixel takes approximately $1.3 \mathrm{sec}$ processing time on a $\mathrm{PC}$ with Pentium III, $933 \mathrm{MHz}$ processor. 


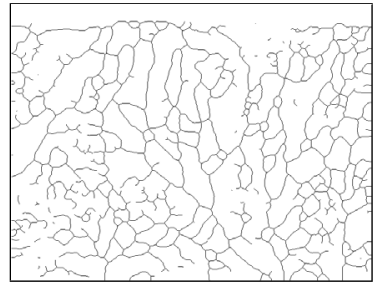

(a)

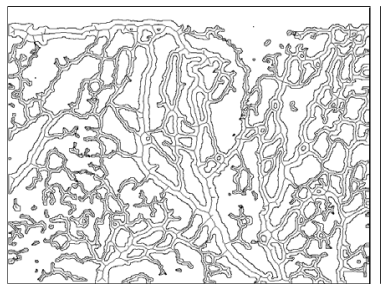

(b)

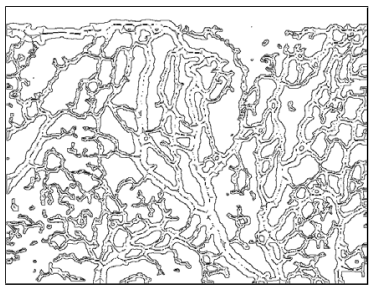

(c)

Fig. 10. Thinning results of Fig. 2 (c): (a) result of mask based method, (b) result of [5], (c) comparison of (a) and (b) (identical points invisible)

\section{Summary}

In this paper we presented a morphological thinning method that consists of iterative conditional parallel erosion. The erosion scheme is defined by a set of mask operations and is combined with a distance map. Due to this combination it is enforced that the skeleton is positioned in the middle of the object. For that reason it combines the advantages of morphological and distance map based methods. The computational time is rather fast and the resulting skeleton image contains only little artefacts. As a result of the last thinning step the skeleton of an object is exactly 1 pixel thin in terms of the 8-topology.

\section{References}

1. B. Steckemetz. Adaptive Skelettierung handgeschriebener Zeichen. In Tagungsband Mustererkennung. DAGM, Springer Verlag, 1997.

2. Donath, K., Wolf, M., Höper, J., Niemann, H., and Plaßwilm, L. (1999). Zeitliche Analyse der Angiogenese im extraembryonalen Gefäßsystem des Hühnerembryos. In Förstner, W., Buhmann, J. M., Faber, A., and Faber, P., Hrsg., DAGM-Symposium, Informatik Aktuell, S. 381-388. Springer. Mustererkennung 1999, 21. DAGMSymposium, Bonn, 15.-17. September 1999, Proceedings.

3. Bernard, T. M. and Manzanera, A. (1999). Improved low complexity fully parallel thinning algorithm. In Proc. Int. Conf. on Image Analysis and Processing (ICIAP), S. 215-220, Venice, Italy. IEEE Computer Society.

4. Manzanera, A., Bernard, T., Prêteux, F., and Longuet, B. (2002). n-dimensional skeletonization: a unified mathematical framework. Journal of Electronic Imaging, 11(1):25-37.

5. Ogniewicz, R.L. and Kübler, O. (1995). Hierarchic voronoi skeletons. In Pattern Recognition 28:343-359. 